\title{
Metabolic Disturbances of a High-Fat Diet Are Dependent on APOE Genotype and Sex
}

\author{
Nahdia S. Jones, Katarina Q. Watson, and \G. William Rebeck \\ https://doi.org/10.1523/ENEURO.0267-19.2019 \\ Department of Neuroscience, Georgetown University, Washington, DC 20007
}

\begin{abstract}
Apolipoprotein E4 (APOE4) is the strongest genetic risk factor for Alzheimer's disease (AD). APOE4 is also associated with an increased risk of metabolic syndrome. Obesity is a major environmental risk factor for AD. While $A P O E$ genotype and obesity independently affect metabolism and cognition, they may also have synergistic effects. Here, we examined the metabolic and behavioral alterations associated with a high-fat diet (HFD) in male and female APOE knock-in mice. Male and female mice were fed a $45 \% \mathrm{kcal} \mathrm{HFD} \mathrm{or} \mathrm{a} 10 \%$ kcal low-fat diet (LFD) for 12 weeks and adipose tissue accumulation, glucose levels, anxiety-like behavior, and spatial memory were examined. We found that with HFD, male APOE4 mice were more susceptible to metabolic disturbances, including visceral adipose tissue (VAT) accumulation and glucose intolerance when compared to APOE3 mice, while female APOE3 and APOE4 mice had similar metabolic responses. Behaviorally, there were no effects of HFD in mice of either genotype. Our results suggest that metabolic responses to HFD are dependent on both sex and APOE genotype.
\end{abstract}

Key words: apolipoprotein E; diet; metabolism; mouse model; obesity

\section{Significance Statement}

Apolipoprotein E4 (APOE4) and obesity are independently associated with increased risk of metabolic syndrome and cognitive impairment. Obesity may cause greater metabolic and cognitive disturbances in APOE4 carriers. However, the metabolic and cognitive effects of obesity on male and female APOE4 carriers remain unknown. Here, we examine and compare the metabolic and cognitive disturbances caused by a high-fat diet (HFD) in both male and female APOE3 and APOE4 mice. Through this study, we examine how HFD affects the APOE3 and APOE4 genotype and how these effects differ across sexes.

\section{Introduction}

Apolipoprotein E4 (APOE4) is the strongest genetic risk factor for Alzheimer's disease (AD; Huang et al., 2004; Raber et al., 2004). In the periphery, APOE is a component of lipoproteins responsible for the metabolism of plasma lipids. Through binding to different lipoprotein receptors, APOE traffics high-density lipoproteins (HDLs) and very low-density lipoproteins (VLDLs) throughout the body for storage or elimination (Huang and Mahley, 2014). In the

\footnotetext{
Received July 9, 2019; accepted September 18, 2019; First published September 25, 2019.

The authors declare no competing financial interests.

Author contributions: N.S.J. and G.W.R. designed research; N.S.J. and K.Q.W. performed research; N.S.J., K.Q.W., and G.W.R. analyzed data; N.S.J. and G.W.R. wrote the paper.
}

CNS, APOE-HDL are responsible for trafficking lipids from astrocytes to neurons and for clearance into the circulation (Liu et al., 2013). There are three APOE alleles, $A P O E 2, A P O E 3$, and APOE4, and each allele is associated with a differential risk of AD. APOE2 has an allele frequency of $8 \%$ in the United States and is associated with a $40 \%$ decreased risk of developing AD (Huang and Mahley, 2014). APOE3 has an allele frequency of $77 \%$; homozygous APOE3 carriers (64\% of the population) are defined as having a normal risk of $\mathrm{AD}$ (Liu et al., 2013). APOE4 has an allele frequency of $15 \%$; heterozygous carriers are 2.3

This work was supported by National Institutes of Health Grants R01 NS100704 and NS100704-S1.

Acknowledgements: We thank the Georgetown Preclinical Imaging Lab for assistance in experimental design and image analysis. 
times more likely to develop $A D$ and homozygous carriers are 14 times more likely (Liu et al., 2013).

Obesity and metabolic syndrome are also major risk factors for AD. Obesity is a medical condition characterized by increased body mass index (BMI) and currently affects $40 \%$ of adults and $20 \%$ of children in the United States (Hales et al., 2017). In the periphery, obesity can lead to metabolic syndrome including increases in visceral adipose tissue (VAT) and subcutaneous adipose tissue (SAT), and decreases in glucose metabolism and insulin sensitivity (Mathieu et al., 2009; Neth and Craft, 2017). In the CNS, obesity is associated with increased inflammation, deficits in cognitive functioning, mild cognitive impairment, and AD (Gustafson et al., 2009; Besser et al., 2014; Bloor and Symonds, 2014).

While $A P O E$ genotype and obesity independently affect $A D$ risk, they may also have combined effects. APOE4 is associated with increased cognitive deficits and increased risk of metabolic syndrome (Arbones-Mainar et al., 2008; Rodriguez et al., 2013; Torres-Perez et al., 2016), which are exacerbated when combined with obesity. Obese APOE4 carriers can have elevated glucose and insulin levels (Elosua et al., 2003), and deficits in cognitive functioning (Ghebranious et al., 2011; Zade et al., 2013). Data in humans is complemented by mouse models. APOE4 knock-in mice have increased insulin resistance and deficits in glucose metabolism when on high-fat diets (HFDs; Arbones-Mainar et al., 2008; Johnson et al., 2017). Cognitive performance of APOE4 mice on HFDs have shown mixed results, with either increased deficits in spatial memory (Johnson et al., 2017) or no cognitive differences (Janssen et al., 2016). Here we compare the effects of a HFD, with macronutrients equivalent to a western diet, on male and female homozygous APOE3 and $A P O E 4$ mice. We examined both metabolic and behavioral alterations and found that HFD increases metabolic disturbances in both $A P O E 3$ and APOE4 mice, with APOE4 mice being more robustly affected. We also found that male and female mice differentially respond to HFD.

\section{Materials and Methods}

\section{Animals/diet}

Male and female human APOE3 and APOE4 knock-in mice on a C57BL/6J background ( $n=5-9 /$ sex; the gift of Patrick Sullivan) were fed either a HFD (45\% kcal fat, Research Diets-D12451) or ingredient matched low-fat diet (LFD; 10\% kcal fat, Research Diets-D12450H) for 12 weeks beginning at six months of age. Food and water were provided ad libitum and mice were weighed weekly during the 12 weeks. At the end of the 12 weeks mice underwent glucose tolerance testing (GTT), abdominal and neck MRI, and behavioral assays which occurred

Correspondence should be addressed to G. William Rebeck at gwr2@georgetown.edu.

https://doi.org/10.1523/ENEURO.0267-19.2019

Copyright (C) 2019 Jones et al.

This is an open-access article distributed under the terms of the Creative Commons Attribution 4.0 International license, which permits unrestricted use, distribution and reproduction in any medium provided that the original work is properly attributed. over a two-week period (12-14 weeks). The mice remained on the diets throughout the GTT, MRIs, and behavioral assays. All experiments followed the guidelines of the Institutional Animal Care and Use Committee.

\section{Glucose testing}

Mice were restricted from food for $6 \mathrm{~h}$ before the measures of baseline glucose levels and glucose tolerance to a glucose bolus. Fasting baseline glucose levels were followed by an intraperitoneal injection of $20 \%$ glucose (1 $\mathrm{mg} / \mathrm{kg}$ ). Blood glucose levels from tail vein withdrawal were measured using the AccuChek Guide glucose meter at $15,30,60$, and 120 min after injection.

\section{MRI}

After completion of behavioral assays, mice underwent small animal imaging in the Preclinical Imaging Research Laboratory at the Georgetown University Medical Center. Mice were anesthetized using 3-5\% isoflurane and maintained with $1-3 \%$ isoflurane. Images were taken with a 7-Tesla horizontal Bruker spectrometer run by Paravision 5.1; body temperature, heart rate, and respiration were monitored throughout the scan. Images were obtained for the abdominal white adipose tissues (WATs) VAT and SAT, and for the neck brown adipose tissue (BAT). Z-stack images were analyzed with ImageJ. VAT and SAT images were quantified as ratios of abdominal adipose tissue to abdominal organs (referred to as "body"). For BAT, images were quantified as the ratio of BAT intensity to the WAT intensity. The BAT and WAT intensities were measured using the mean gray value in ImageJ, with the darker areas being reflected as higher mean gray values indication higher BAT intensities. Higher BAT intensity indicates more BAT, which has the ability to convert excess food energy into thermal energy (Schulz and Tseng, 2013).

\section{Behavioral assays: open field test (OFT), elevated zero maze (EZM), and Barnes maze (BM)}

For all behavioral assays, mice were placed in the behavioral suite for a 30-min acclimation period.

\section{OFT}

Mice freely explored a square $(43 \times 43 \times 30 \mathrm{~cm})$ open field apparatus for $300 \mathrm{~s}$. During free exploration, locomotor activity and anxiety-like behaviors were recorded. The apparatus was divided into an inner zone and a bordering outer zone that lined the apparatus's walls. Mice were placed in the center of the inner zone and behavior was recorded for the duration of the test. Behavior was recorded with Med Associates Activity Monitor 7. For locomotion, average speed $(\mathrm{m} / \mathrm{s})$ was assessed. For anxiety-like behavior, time spent in the inner versus outer zone $(9 \times 9 \mathrm{~cm})$ was assessed as increased time in the outer zone indicating increased anxiety (Seibenhener and Wooten, 2015). Data were analyzed with GraphPad Prism 8.

\section{EZM}

Mice were exposed to a circular elevated zero apparatus $(50 \mathrm{~cm}$ from floor, $50 \mathrm{~cm}$ in diameter, and $15-\mathrm{cm}$ high closed regions) for $300 \mathrm{~s}$ of free exploration. The apparatus consists two closed regions and two open regions of 

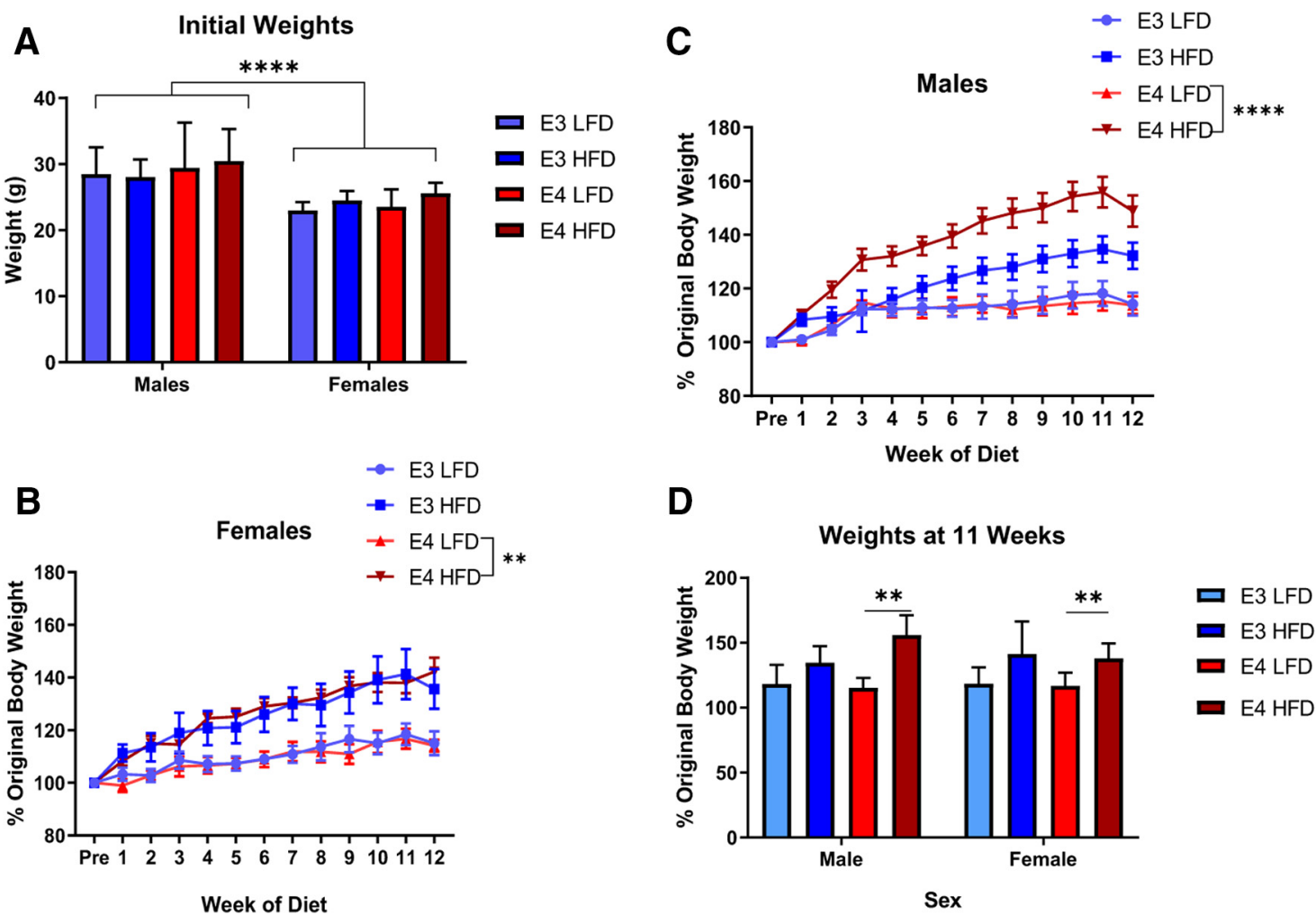

Figure 1. HFD increases weight gain and glucose intolerance. Weight gain comparison from pre-diet to week 12 on diet across $A P O E$ genotypes and sex. Initial weights of all mice in grams $(\boldsymbol{A})$. Diet associated weight gain of female $A P O E 3$ and $A P O E 4$ mice $(\boldsymbol{B})$ and male $A P O E 3$ and $A P O E 4$ mice $(\boldsymbol{C})$. Direct comparison of male and female weight gain at week 11 on diet (D). E3 LFD: light blue, APOE3 mice on a LFD, E3 HFD: dark blue, APOE3 mice on a HFD, E4 LFD: light red, APOE4 mice on a LFD, E4 HFD: dark red, APOE4 mice on a HFD. Three-way ANOVA Tukey's multiple comparison test, $N=5-9$. $\boldsymbol{A}, * * * * p<0.0001 ; \boldsymbol{B}, * * p<0.003 ; \boldsymbol{C}, * * * * p<0.0001$; $\boldsymbol{D}, * * p<0.0046$.

equal sizes. Mice were placed on the center of an open region to begin testing and behavior was recorded for the duration of the 300 s using ANY-maze Behavioral tracking software 6.0. Time spent in the closed versus open regions of the apparatus was examined as a measure of anxiety and willingness to explore. Data were analyzed with GraphPad Prism 8.

\section{$B M$}

Mice were exposed to the BM for five consecutive days to test spatial learning and memory, as described (Speidell et al., 2019). The maze was present in a brightly lit room and $90-\mathrm{dB}$ white noise. Mice were habituated to the maze on day 1 , and then had four consecutive training days. During training days, mice underwent four trials with 15 min between each trial, and latency to first nose poke and latency to enter the escape hole (latency to escape) were recorded to examine spatial memory. Mice were recorded with ANY-maze Behavioral tracking software 6.0, and data were analyzed with GraphPad Prism 8.

\section{Statistics}

All data are expressed as mean \pm SD with the exception of behavioral assays which are expressed as mean \pm SE. Comparisons between genotype, sex and diet were analyzed by three-way ANOVAs with Tukey's multiple comparison test. Comparisons between genotype and sex were analyzed by two-way ANOVAs with Sidak's multiple comparison test. Statistical significance was determined by a probability error of $p<0.05$. All analyses were done using GraphPad Prism 8.

\section{Results}

\section{HFD increases the weight of $A P O E 3$ and APOE4 mice}

To examine how the different $A P O E$ genotypes respond to obesity, we used a diet induced obesity model. Male and female mice (six months old) were placed on a HFD for 12 weeks and weighed weekly. At six months old, the male mice (across genotypes) weighed significantly more than female mice $(p<0.0001$; Fig. $1 A)$. To directly compare weight gain trajectories, weights were calculated as a percentage of each mouse's original body weight. All groups gained weight over the course of the experiment, with HFD groups gaining more weight compared to LFD groups (Fig. 1).

In female mice, HFD resulted in a $40 \%$ increase from original body weight by week 11 in both $A P O E 3$ and APOE4 mice $(p<0.0001)$; both genotypes gained weight at the same rate. HFD mice also weighed more than LFD mice (APOE3: $p<0.06$, APOE4: $p<0.005$ ). Female $A P O E 3$ and $A P O E 4$ mice on the LFD experienced slight weight gain $(15 \%, p>0.8$; Fig. 1B). 
In male mice, HFD resulted in a $30 \%$ increase from original body weight in APOE3 mice by week 11 ( $p<$ 0.0001 ) and resulted in a $45 \%$ increase from original body weight in APOE4 mice by week 11 ( $p<0.0001)$, although the differences between APOE3 and APOE4 genotypes were not statistically significant $(p=0.15)$. APOE3 and $A P O E 4$ mice on the LFD experienced slight weight gain $(17 \%, p>0.8$; Fig. 1C). HFD APOE3 mice did not weigh significantly more than the LFD APOE3 mice $(p=0.57)$; HFD APOE4 mice did weigh significantly more than LFD APOE4 mice $(p<0.0001)$.

Across sexes, the weight gain due to HFD did not differ. However, while male APOE3 did respond to HFD, they gained $15 \%$ less weight than male APOE4 mice or female mice (Fig. 1D). On week 12, there was a slight decrease in body weight associated with the beginning of the metabolic and behavioral assays; therefore, statistical tests were conducted on data from week 11.

\section{HFD increases baseline glucose levels and glucose intolerance in $A P O E 3$ and $A P O E 4$ mice}

Deficits in glucose metabolism are also associated with HFD. These deficits can lead to Type II diabetes and cognitive deficits. To test whether our model alters glucose metabolism, baseline glucose levels and glucose tolerance were measured after 12 weeks of HFD (Fig. 2).

In females, HFD APOE3 and APOE4 mice had similar baseline glucose levels; these levels did not differ from LFD APOE3 and APOE4 mice. In males, HFD APOE3 and $A P O E 4$ mice had similar baseline glucose levels; however, their levels were elevated when compared to LFD APOE3 and APOE4 mice ( $p<0.002$; Fig. $2 A)$. Across sexes, male HFD mice had significantly higher baseline glucose levels than the female HFD mice $(p<0.0001$; Fig. $2 A)$. We reasoned that increased baseline glucose may be associated with weight gain, given the disparate levels of weight gains in male versus female mice. To test this hypothesis, we determined the correlation of weight gain with baseline glucose across genotype and sex. APOE3 and $A P O E 4$ weight gain was positively correlated with increased baseline glucose in males, but not females (APOE3: $p=0.001, R^{2}=0.68$, APOE4: $p=0.003, R^{2}=$ 0.62; Fig. $2 B$ ).

After baseline glucose levels, mice underwent GTT as a measurement of glucose metabolism. A bolus of glucose was given, and glucose levels measured at $15,30,60$, and $120 \mathrm{~min}$. In females, when compared to baseline, there was an increase in glucose levels in the first $15 \mathrm{~min}$ in all groups $(p<0.003)$. This increase remained in the HFD groups at $30 \mathrm{~min}(p<0.0001)$, and $60 \min (p<0.0001$; Fig. 2C). In males, when compared to baseline, there was an increase in glucose levels in the first $15 \mathrm{~min}$ and remained elevated at $30 \mathrm{~min}$ in all groups $(p<0.02)$. At 60 $\mathrm{min}$, all mice returned to the range of baseline glucose except for HFD APOE4 mice $(p<0.002$; Fig. $2 D)$. This indicates that the HFD APOE4 mice did not metabolize the glucose as quickly or efficiently as the HFD APOE3 mice or the LFD APOE4 mice.

To examine overall differences in glucose tolerance over time across genotype and sex, we analyzed area under the curve in the GTT. In females, HFD APOE4 mice had a larger deviation in glucose than HFD APOE3 mice ( $p$ $<0.0003$ ) and LFD APOE4 mice had a larger deviation in glucose than LFD APOE3 mice $(p<0.02)$. HFD APOE4 mice also had a larger deviation than LFD APOE4 mice ( $p$ $<0.02$ ). This difference was not seen when comparing HFD APOE3 mice and LFD APOE3 mice (Fig. 2E). In males, HFD APOE4 mice had a larger deviation in glucose than HFD APOE3 mice $(p<0.0001)$. HFD mice also had a larger deviation in glucose than both LFD APOE3 mice $(p<0.05)$, and APOE4 mice ( $p<0.0001$; Fig. 2E). Across sexes, glucose deviations in male HFD APOE4 mice were greater than deviations seen in all female groups $(p<$ 0.0003). Deviations in male HFD groups were larger than deviations seen in the female groups except female HFD APOE4 mice ( $p<0.003$; Fig. $2 E$ ).

To test whether the glucose intolerances found could result from weight gains, we ran correlational analyses comparing weight gain with glucose levels $30 \mathrm{~min}$ after bolus. In both $A P O E 3$ and APOE4 mice, an increase in weight was positively correlated with higher glucose levels $(p<0.007)$, indicating any increase in weight may strongly affect glucose intolerance. There was a stronger positive correlation between weight gain and glucose intolerance in APOE3 mice $(p=0.01)$ indicating weigh gain can drive glucose intolerance in $A P O E 3$ mice while $A P O E 4$ mice are more susceptible to glucose intolerance at lower weights (Fig. 2F). Glucose levels were significantly correlated with weight gain regardless of sex with the exception of APOE4 males $(p<0.02$; Fig. $2 F)$.

\section{HFD increases VAT and SAT in APOE3 and APOE4 mice}

A metabolic disturbance associated with HFD is increased adipose tissue. SAT is the adipose tissue more associated with obesity; however, VAT is more noxious due to its direct contact with the organs and its ability to release inflammatory cytokines (Hotamisligil et al., 1995). To test whether our model results in increases in specific types of adipose tissue, we used small rodent MRls to examine both VAT and SAT levels (Fig. $3 A$ ).

In females, HFD caused an increase in VAT compared to LFD $(p<0.0001 ;$ Fig. $3 B$ ). In males, HFD APOE4 mice accumulated more VAT than LFD APOE4 mice $(p<$ $0.0001)$, but there was no similar effect for APOE3 mice ( $p$ $=1.0$; Fig. $3 B$ ). Across sexes, HFD mice had similar elevated VAT levels, except for the male APOE3 mice, which did not differ from LFD mice $(p<0.02$; Fig. $3 B$ ).

We analyzed the correlation between VAT and GTT, including the possible effects of genotype, sex, and diet. There was no correlation between VAT and GTT when considering genotype and diet (Fig. $3 C$ ); however, there was a correlation between VAT and GTT only in male APOE4 mice $\left(R^{2}=0.6, p=0.03\right.$; Fig. $\left.3 C\right)$. We also ran correlational analyses comparing weight gain and VAT to see whether VAT was a large contributor to the weight gain. VAT and weight gain in APOE3 females and APOE4 mice positively correlated (APOE3: $R^{2}=0.52 p=0.005$, APOE4: $\left.R^{2}=0.40, p=0.01\right)$; however, there was not a positive correlation between VAT and weight gain in 
A

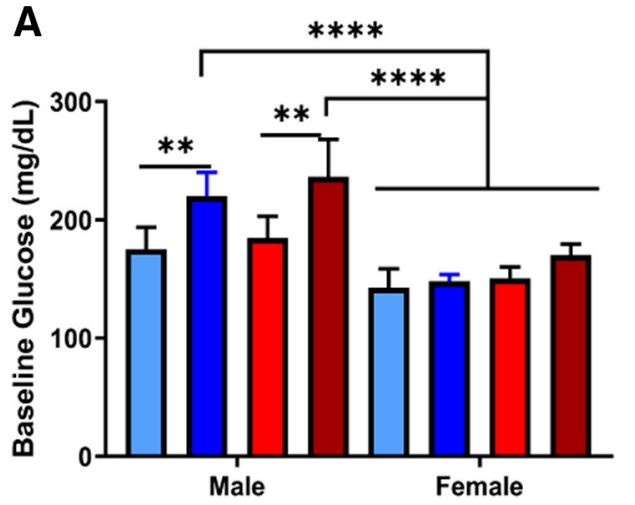

C

\section{Females}

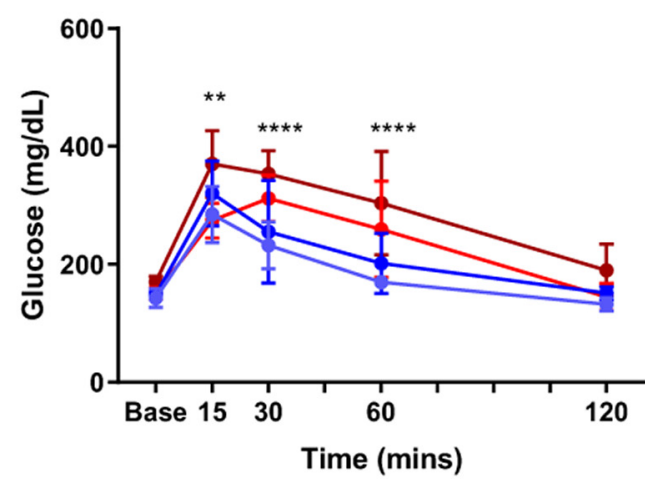

E

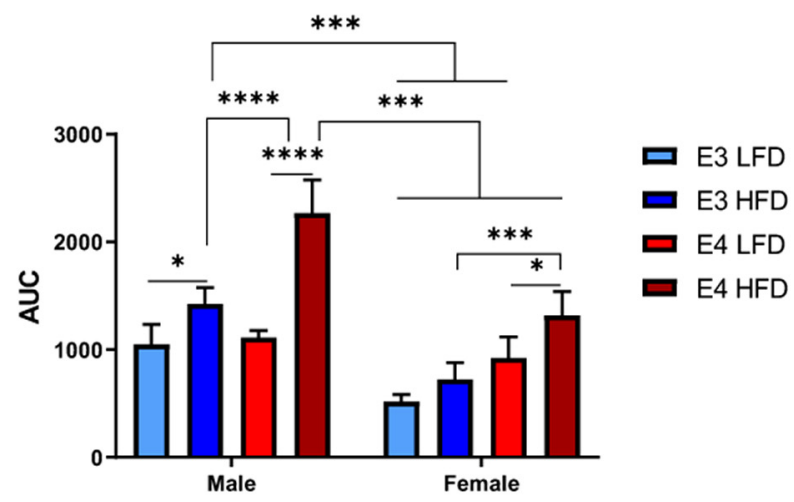

B

- E3 Female

- E3 Male **

- E4 Female

$\rightarrow$ E4 Male **

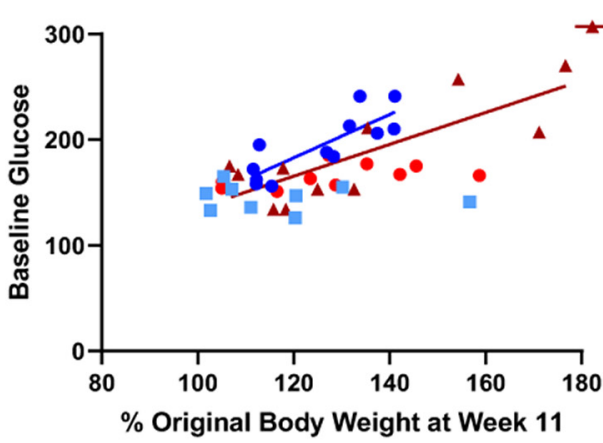

D Males

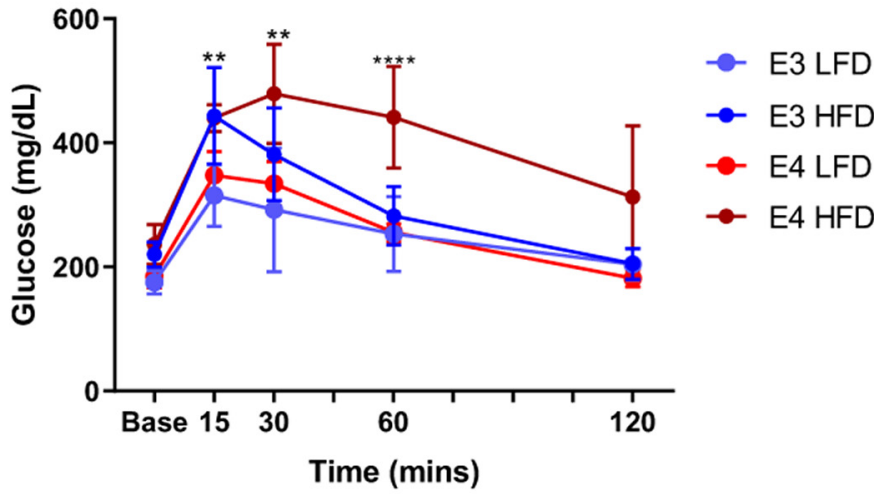

$\mathbf{F}$

Correlation-Weight and Glucose

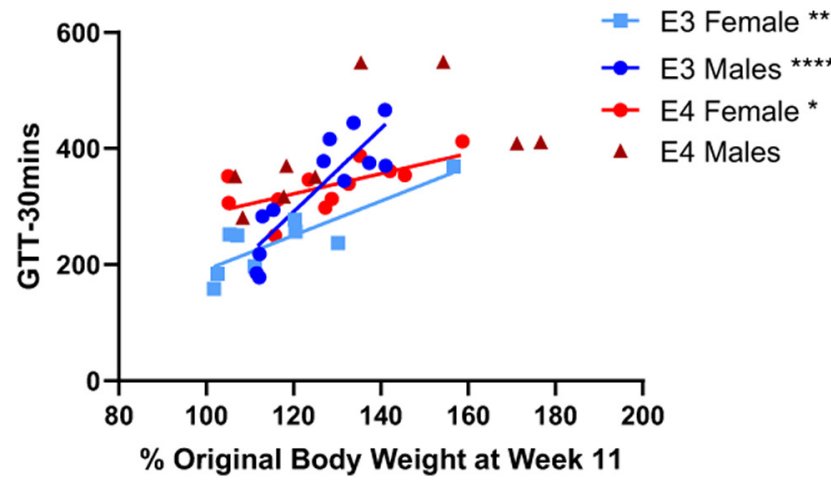

Figure 2. HFD increases baseline glucose and glucose intolerance. Comparison of baseline glucose levels in male and female APOE3 and APOE4 mice, first comparing within sex differences then across sex differences $(\boldsymbol{A})$. Correlational analyses between weight, glucose levels, and sex. Lines indicate significant correlations $(\boldsymbol{B})$. GTT in female $(\boldsymbol{C})$ and male $(\boldsymbol{D})$ APOE3 and $A P O E 4$ mice. Area under the curve (AUC), complete deviation from baseline glucose levels, comparing within sex differences and across sex differences (E). Correlation between genotype, weight gain, sex, and glucose levels at $30 \mathrm{~min}$. Lines indicate significant correlations $(\boldsymbol{F})$. $\boldsymbol{A}, \boldsymbol{C}-\boldsymbol{E}, N$ $=4-6$. Three-way ANOVA Tukey's multiple comparison test. $\boldsymbol{A}, * * p<0.002 ; * * * * p<0.0001 ; \boldsymbol{C}, * * p<0.003$ all groups deviate from baseline at $15 \mathrm{~min}, * * * * p<0.0001 \mathrm{HFD}$ and LFD APOE4 deviates from baseline at 30 and $60 \mathrm{~min} ; \boldsymbol{D}, * * p<0.002$ all groups deviate from baseline at $15 \mathrm{~min}, * p<0.02$ all groups deviate from baseline at $30 \mathrm{~min}, * * * p<0.0002$ HFD APOE4 deviates from baseline at $60 \mathrm{~min} ; \boldsymbol{E}, * p<0.02, * * p<0.01, * * * p<0.0003, * * * * p<0.0001$. $\boldsymbol{B}, \boldsymbol{F}$, Linear regression. $\boldsymbol{B}, A P O E 3$ males $(N=12): R^{2}=0.68, p$ $=0.001$. APOE4 males $(N=11): R^{2}=0.63, p=0.004$. $\boldsymbol{F}$, APOE3 males $(N=12): R^{2}=0.75, p=0.0002$. APOE3 females $(N=9)$ : $R^{2}=0.72, p=0.004$. APOE4 females $(N=12): R^{2}=0.42, p=0.02$. 

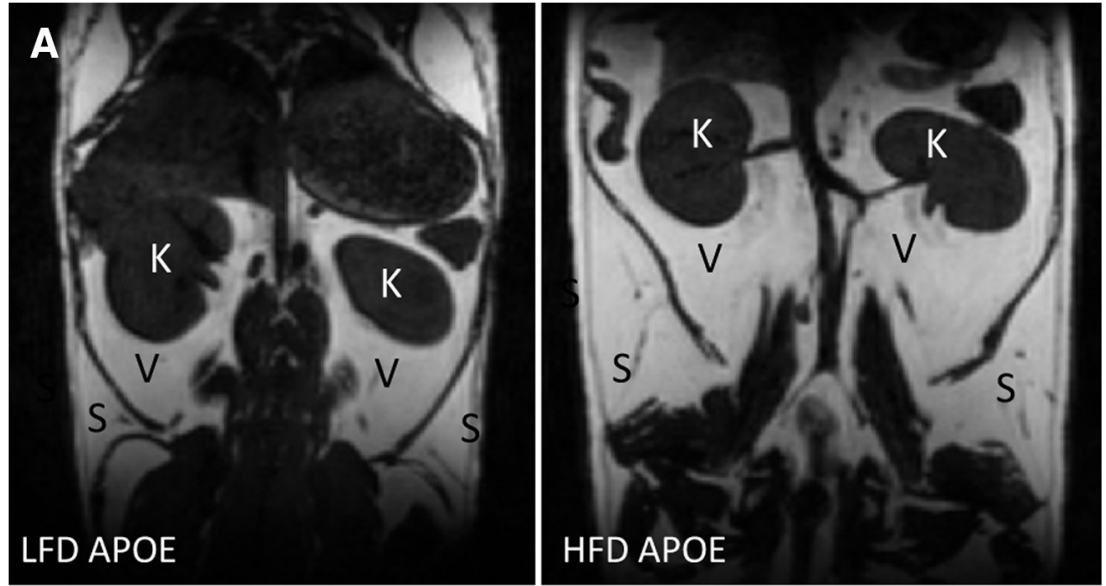

B 므 E3 LFD

E3 HFD

마 LFD

마 HFD

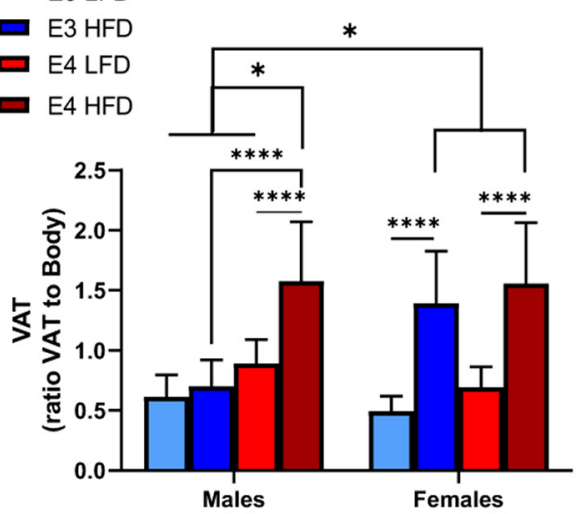

C

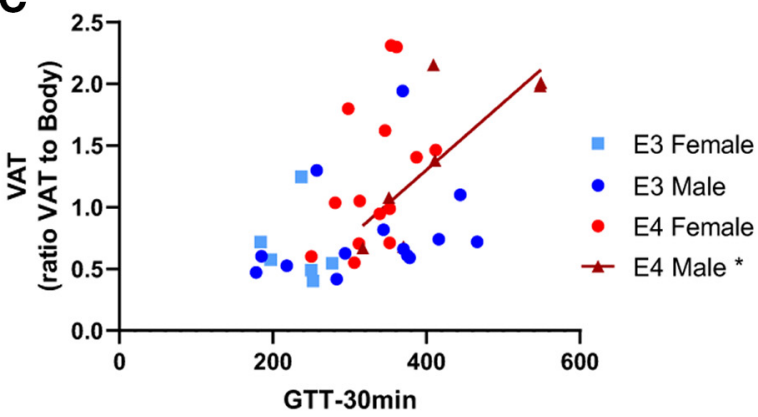

D

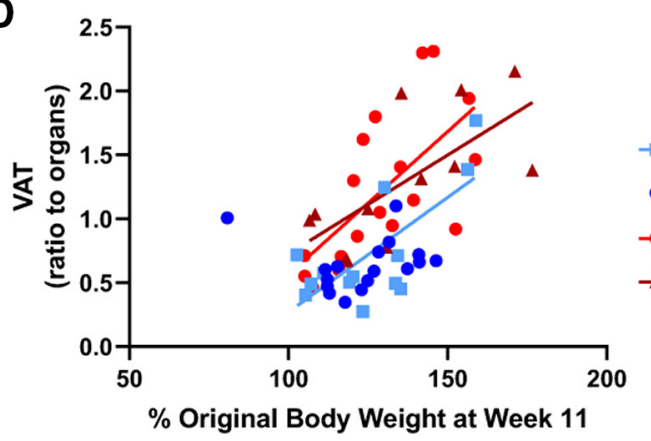

Visceral Adipose Tissue

Figure 3. HFD increases VAT in APOE3 and APOE4 mice. Representative image of VAT and SAT in LFD mouse and HFD mouse. S, SAT; V, VAT; K, kidneys (A). Within sex and across sex quantification of VAT in APOE3 and APOE4 mice (B). Correlation of glucose intolerance and VAT accumulation across $A P O E$ genotypes and across sex. Lines indicate significant correlations (C). Correlation of weight gain and VAT accumulation across APOE genotypes and across sex. Lines indicate significant correlations $(\boldsymbol{D})$. $\boldsymbol{B}, N=5-9$. Within sex comparison: $* * * * p<0.0001$, three-way ANOVA Tukey's multiple comparison test. Across sex comparison: $* p<0.02$, three-way ANOVA Tukey's multiple comparison test. $\boldsymbol{C}, \boldsymbol{D}$, Linear regression analyses. $\boldsymbol{C}, A P O E 3$ female $(N=6): R^{2}=0.52, \mathrm{p}=$ 0.005; APOE4 male $(N=7): R^{2}=0.48, p=0.01$; APOE4 female $(N=14): R^{2}=0.43, p=0.003$. $\boldsymbol{D}$, APOE4 male $(N=14): R^{2}=0.62$, $p=0.03$.

APOE3 males (Fig. 3D). These findings indicate that VAT may act as a contributor to weight gain and glucose intolerance.

The effects of HFD on SAT mirrored its effects on VAT. In females, HFD APOE3 and APOE4 mice had similar levels of SAT, and HFD caused an increase in SAT compared to LFD. $(p<0.03)$. In males, SAT accumulation did not differ across genotype or diet (Fig. 4A). VAT and SAT levels strongly correlated $\left(R^{2}=0.47, p<0.0001\right.$; Fig. $\left.4 B\right)$.

\section{Sex affects BAT intensity in APOE3 and APOE4 mice}

BAT is a metabolically active adipose tissue (Schulz and Tseng, 2013). To examine diet associated BAT alterations, we used small rodent MRI and imaged neck BAT (Fig. 5A).
A

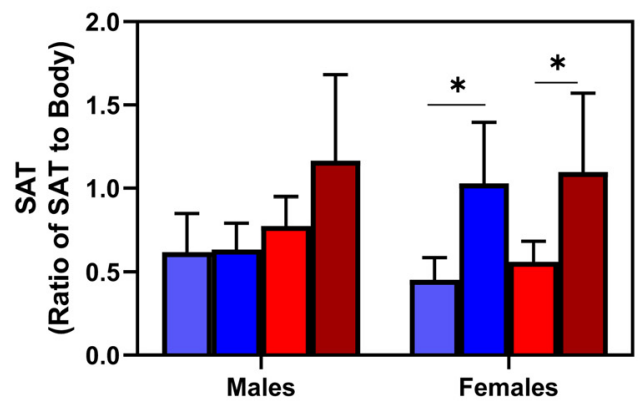

B

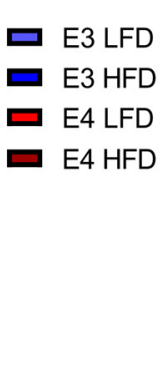

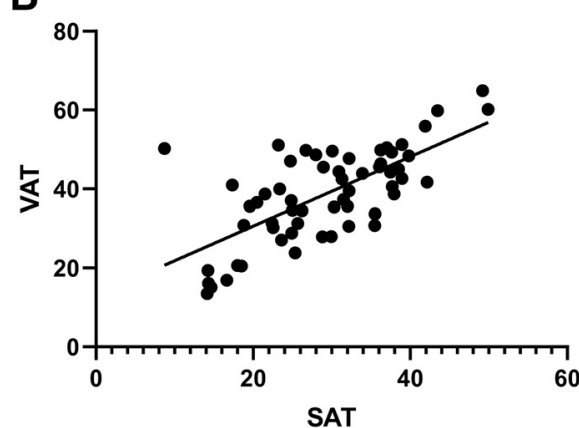

Figure 4. HFD increases SAT in APOE3 and APOE4 mice. Within sex and across sex quantification of VAT in APOE3 and APOE4 mice (A). Correlation of VAT and SAT accumulation (B). $\boldsymbol{A}, N=5-9, * p<0.04$, three-way ANOVA Tukey's multiple comparison test. $\boldsymbol{B}, \boldsymbol{N}$ $=59$, linear regression $R^{2}=0.47, p \leq 0.0001$. 

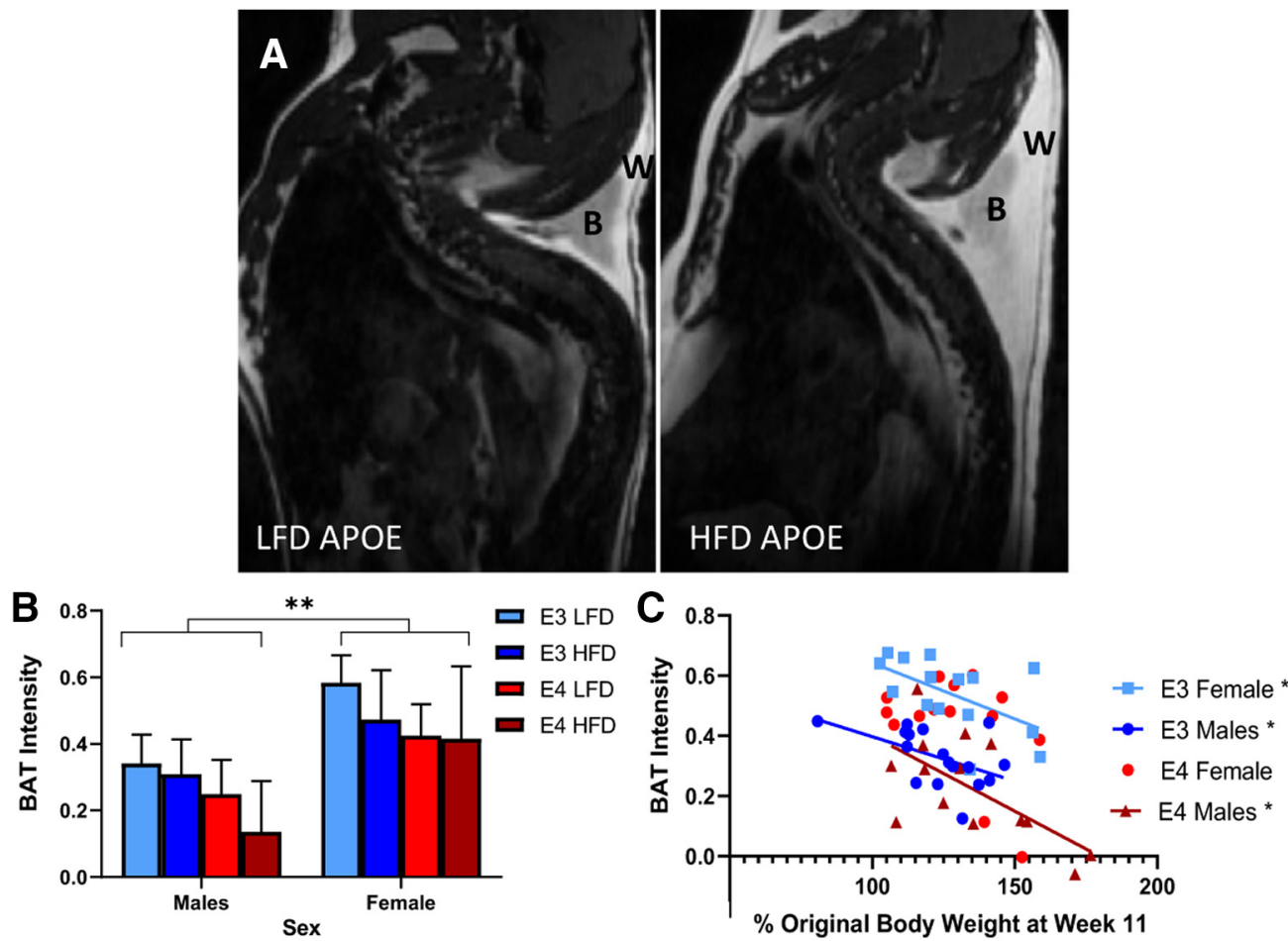

Figure 5. Male APOE mice have decreased BAT. Representative image of BAT in the neck in a LFD mouse and HFD mouse (A). Comparison of BAT intensity in male and female APOE3 and APOE4 mice (B). Correlation of genotype and weight to BAT intensity then sex and weight to BAT intensity lines indicate significant correlations (C). B, BAT; W, WAT; S, spine, TAT, total adipose tissue; $\boldsymbol{B}, N=5-9, * * p<0.004$, three-way ANOVA Tukey's multiple comparison test. $\boldsymbol{C}$, Linear regression. $A P O E 3$ male $(N=17): R^{2}=0.25$, $p=0.04 ;$ APOE3 female $(N=15): R^{2}=0.32, p=0.03 ;$ APOE4 male $(N=14): R^{2}=0.42, p=0.01$.

We examined intensity of BAT, with decreasing intensities indicating the transition to WAT. There was no effect of diet on BAT; however, there were sex differences. Male mice had significantly lower BAT intensities than female mice $(\sim 30 \%, p<0.004$; Fig. $5 B)$. The lower BAT intensities indicate less thermogenic energy expenditure which has been implicated in decreased resistance to diet induced obesity (Schulz and Tseng, 2013). We ran correlational analyses to see whether BAT intensity individually correlated with weight gain. In male APOE4 mice, there was a negative correlation between weight gain and BAT intensity $\left(R^{2}=0.42, p=0.01\right.$; Fig. $\left.5 C\right)$. This was also seen in APOE3 mice $\left(R^{2}=0.3, p=0.03\right.$, Fig. $\left.5 C\right)$. These correlations indicate weight gain can directly decrease BAT levels, particularly in APOE3 and male APOE4 mice.

\section{Effects of HFD on behavior in APOE3 and APOE4 mice}

We tested the effects of $A P O E$ genotype, sex, and diet on cognitive domains in these mice. Since HFD resulted in significant weight gain, we first examined whether movement had been impaired. In the OFT, there were no differences in average speed regardless of diet, sex, or genotype (Fig. 6A).
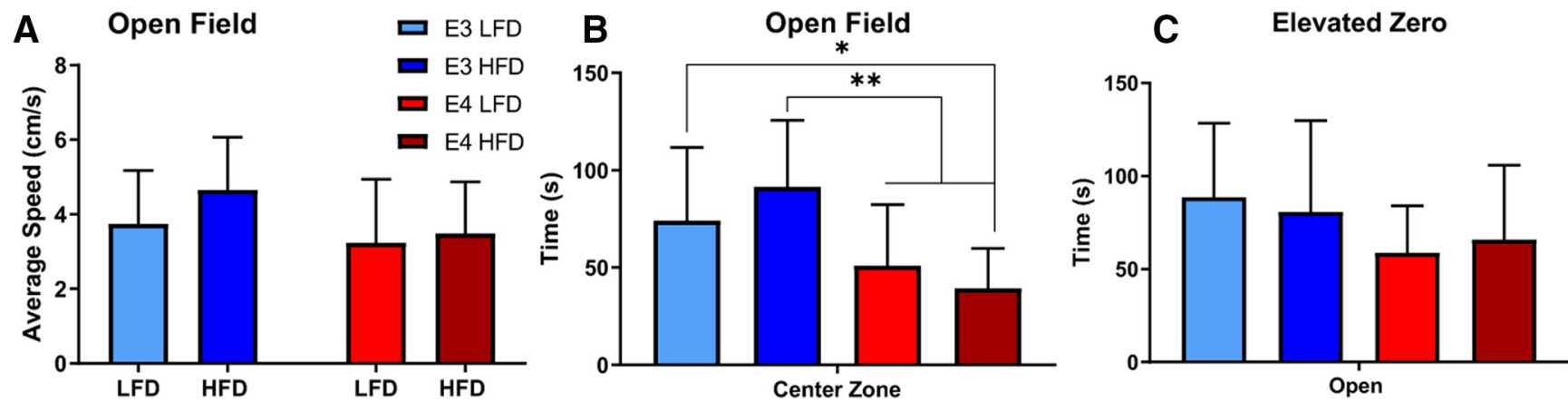

Figure 6. Effects of diet on locomotor activity and anxiety-like behavior. Mice were placed on an open field apparatus and locomotion was recorded. Average speed on OFT analyzed by APOE genotype and diet $(\boldsymbol{A})$. Mice were placed on the open field apparatus and EZM and anxiety-like behavior were analyzed. Time spent in the center zone of the OFT $(\boldsymbol{B})$. Time spent in the open arms of the EZM (C). $\boldsymbol{A}-\boldsymbol{C}, \boldsymbol{N}=13-15$ mice. Two-way ANOVA Sidak's multiple comparison test. $\boldsymbol{B}, * p<0.05, * * p<0.002$. 

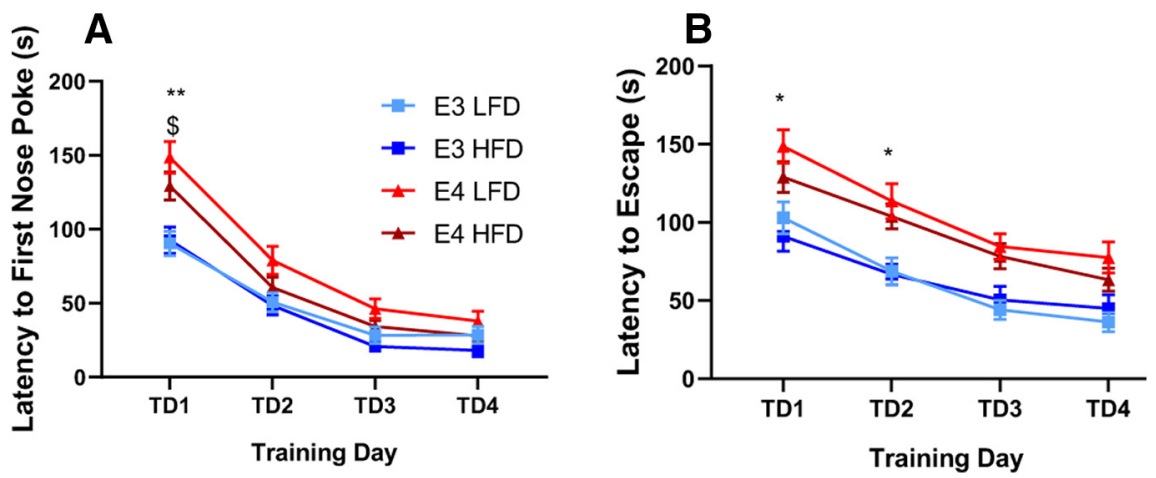

Figure 7. Effects of diet on BM performance. Mice were trained on the BM for four consecutive days, and memory acquisition was measured. Latency to first nose poke of the escape hole $(\boldsymbol{A})$. Latency to escape from the BM (B). $\boldsymbol{A}, \boldsymbol{B}, N=13-15 ; * p<0.03 ; \$ p<$ $0.05, * * p<0.004$, two-way ANOVA, Sidak's multiple comparison test.

To examine whether HFD induced cognitive alterations in this experiment, we used the OFT, EZM, and BM. OFT and EZM were both used to measure anxiety like behavior. For the OFT, time spent in the center zone was used as a measure of decreased anxiety. We found that all APOE4 mice spend less time in the center zone than HFD APOE3 mice $(* p<0.05, * * p<0.002$; Fig. $6 B)$. There were no differences between sexes (data not shown). EZM, a second measure of anxiety like behavior, did not show any differences by diet, APOE genotype, or sex (Fig. 6C).

We used the BM to test spatial learning and memory. The mice were exposed to the maze for four training days and latency to first nose poke and latency to escape were measured each day. For latency to first nose poke, APOE4 mice showed less learning on training day 1 , but matched APOE3 mice by training day $2(p<0.03$; Fig. $7 A)$. For latency to escape, APOE4 mice were delayed for the first two training days, but by training day 3 the latency to escape matched APOE3 mice $(p<0.03$; Fig. $7 B)$. There was no effect of diet on either APOE3 or APOE4 groups.

\section{Discussion}

Although not as severe as the risk of $A D$ in homozygous APOE4 carriers, metabolic disturbances caused by a HFD can have a 2- to 3-fold increased risk of cognitive impairment and AD (Gunstad et al., 2007; Whitmer et al., 2008; Profenno et al., 2010). Using an APOE knock-in mouse model, we found that $A P O E 4$ increases metabolic disturbances in response to HFD. Furthermore, sex plays a pivotal role in the effects of HFD. We examined differences in weight, baseline glucose levels, glucose intolerance, and adipose tissue disposition and found these to be the most significantly increased in male APOE4 mice. Female $A P O E 3$ and $A P O E 4$ mice responded similarly to HFD with increased weight, glucose intolerance, and adipose tissue levels. In terms of the types of adipose tissue that increased under the HFD, in males, VAT increases were seen in the APOE4 mice, but not APOE3 mice. SAT increases were not seen in $A P O E 3$ or $A P O E 4$ mice. These findings demonstrate that the male APOE4 group has the greatest accumulation of VAT in response to HFD. In females, VAT and SAT increases were seen in both $A P O E 3$ and $A P O E 4$ mice in response to HFD, indicating there is a similar accumulation in both types of adipose tissue. Throughout the study, female mice had similar metabolic responses to HFD regardless of $A P O E$ genotype and male APOE4 mice had more robust metabolic disturbances.

While we cannot directly compare our study to previous studies due differences in the age of mice, diet composition, and length of time on diets, there are similarities across models. With wild-type mice on HFD, male and female mice accumulate similar levels of VAT, but male mice display higher fasting blood glucose levels, insulin levels, and insulin resistance (Macotela et al., 2009; Hwang et al., 2010; Medrikova et al., 2012; Barron et al., 2013). Human studies also showed this pattern: males have increased chances of metabolic syndrome associated with obesity (Hadaegh et al., 2013; Pradhan, 2014). These studies demonstrate that the rodent models can reflect well some effects of HFD on human metabolic disturbances.

Several studies have been conducted in APOE mice on the effects of HFDs, because clinical studies showed APOE4-positive individuals have increased risk of metabolic syndrome (Arbones-Mainar et al., 2008; TorresPerez et al., 2016) and obese APOE4 carriers have increased metabolic disturbances when compared to APOE3 carriers (Elosua et al., 2003). Diverse studies, including ours, showed that there were no differences in baseline glucose levels between APOE3 and APOE4 mice on HFD, and that HFD induced worse glucose tolerance in APOE4 mice than in APOE3 mice (Table 1). These findings support the observed susceptibility of human APOE4 carriers to metabolic disturbances, underscoring the importance of diet for APOE4 individuals in particular.

There are several findings that differ from our work (Table 1). Published studies show that APOE3 mice on several types of HFDs gain more weight when compared to APOE4 mice (Arbones-Mainar et al., 2008; Huebbe et al., 2015; Johnson et al., 2017), and APOE3 mice have greater VAT accumulation (Arbones-Mainar et al., 2008; Johnson et al., 2017). In all studies the VAT accumulation reflects the weight gain, with the heavier groups having larger VAT compositions. These differences in VAT accumulation and weight gain across studies could be due to different diet compositions. Our study uses a lard based 
Table 1. Studies of the effects of a HFD on APOE3 and APOE4 mice

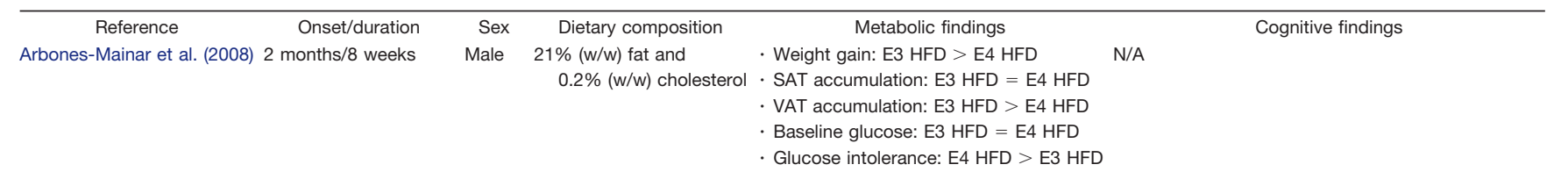

\begin{tabular}{|c|c|c|c|c|}
\hline To et al. (2011) & 3 months $/ 32$ weeks & Male & $60 \% \mathrm{kcal}$ fat from lard & $\begin{array}{l}\text { - Baseline glucose: E3 HFD = E4 HFD } \\
\text { - Glucose intolerance: E3 HFD = E4 HFD }\end{array}$ \\
\hline Huebbe et al. (2015) & $6-8$ weeks/8 months & Female & $21 \%$ fat from milk & - Weight gain: E3 HFD > E4 HFD \\
\hline Arbones-Mainar et al. (2016) & 2 months/1-10 months & Male & $\begin{array}{l}21 \%(\mathrm{w} / \mathrm{w}) \text { fat from } \\
\text { milk and } 0.2 \%(\mathrm{w} / \mathrm{w}) \\
\text { cholesterol }\end{array}$ & $\begin{array}{l}\text { - Weight gain: E3 HFD > E4 HFD } \\
\text { - Baseline glucose: E3 HFD = E4 HFD }\end{array}$ \\
\hline Johnson et al. (2017) & 9 months $/ 6$ months & Female & $60 \% \mathrm{kcal}$ fat from lard & $\begin{array}{l}\text { - Weight gain: E3 HFD > E4 HFD } \\
\text { - VAT accumulation: E3 HFD > E4 HFD } \\
\text { - Baseline glucose: E3 HFD = E4 HFD } \\
\text { - Glucose intolerance: E4 HFD > E3 HFD }\end{array}$ \\
\hline Johnson et al. (2019) & 9 months $/ 6$ months & Female & $60 \% \mathrm{kcal}$ fat from lard & $\begin{array}{l}\text { - Weight gain: E3 HFD > E4 HFD } \\
\text { - SAT accumulation: E3 HFD = E4 HFD } \\
\text { - VAT accumulation: E3 HFD > E4 HFD } \\
\text { - Baseline glucose: E3 HFD = E4 HFD } \\
\text { - Glucose intolerance: E3 HFD = E4 HFD }\end{array}$ \\
\hline
\end{tabular}

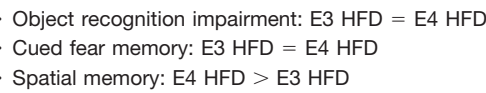

$45 \%$ kcal fat diet; other studies use either a diet where the fat is composed of milk (Arbones-Mainar et al., 2008, 2016; Huebbe et al., 2015) or $60 \%$ kcal fat from lard (To et al., 2011; Johnson et al., 2017, 2019). These findings raise the interesting possibility that both the components and the percentage of fat can differentially affect weight gain in APOE4 carriers. In humans, healthy APOE4 carriers have lower BMI (Tejedor et al., 2014), although they remain more susceptible to metabolic and cognitive disturbances.

Studies on the effects of HFDs on cognition in nonAPOE mice showed spatial memory deficits and deficits in other cognitive task including novel object recognition and fear condoning in wild type mouse models (Hwang et al., 2010; Kesby et al., 2015) and AD mouse models (Barron et al., 2013; Knight et al., 2014; Kesby et al., 2015; Lin et al., 2016; Johnson et al., 2017). Studies on the effects of diet on cognition in $A P O E$ mice showed either equal levels of impairment in $A P O E 3$ and $A P O E 4$ mice on HFD or increased impairment in APOE4 mice on HFD depending on the behavioral assay (Johnson et al., 2017, 2019). We did not observe robust behavioral effects with our behavioral assays. APOE4 mice exhibited more anxiety like behavior on the OFT but not on the EZM. With the $\mathrm{BM}, A P O E 4$ mice had impairment in spatial learning overall, but diet had only an effect on TD1. Potential effects of diet here may have been obscured by sex differences, which could be addressed in larger cohorts. Previous studies have shown APOE4 mice have cognitive deficits or decreased neuronal complexity from as early as three months and these deficits remain at later ages such as 21 months (Bour et al., 2008; Rodriguez et al., 2013; Speidell et al., 2019), consistent with APOE genotype dependent deficits seen in our study. Performance in cognitive tasks have differed between sexes also with females performing worse than males (Bour et al., 2008), further emphasizing the need for these behavioral assays to be replicated with a greater number of animals across sexes.

In humans, obesity has been linked to increased risk of $A D$, cognitive disturbances, and decreases in structural integrity (Enzinger et al., 2005; Gunstad et al., 2007). Middle aged obesity is particularly impactful, associated with increased risk of cognitive disturbances and dementia (Whitmer et al., 2005; Gustafson et al., 2007; Tolppanen et al., 2014). However, higher BMI at later ages is protective (Tolppanen et al., 2014), highlighting a complex relationship between $\mathrm{BMI}$ and cognition. Interestingly, while obesity in males is associated with increased susceptibility to metabolic disturbances, obesity in females is associated with increased susceptibility to cognitive changes (Elias et al. 2005; Moser and Pike 2016). Obese females compared to obese males have increased risk of $\mathrm{MCl}$ leading to $\mathrm{AD}$, decreased cognitive performances, decreased structural brain integrity (Moser and Pike, 2016). APOE4 females (compared to APOE4 males) have an equivalent risk of $A D$, with a significantly earlier age of onset between 65 and 75 years old (Neu et al., 2017). In mouse studies of $A P O E$ mice crossed with 5xFAD (EFAD), male obese E4FAD mice have higher levels of beta amyloid deposits, glial reactivity, and inflammatory markers compared to non-obese E4FAD mice or obese E3FAD mice (Moser and Pike, 2017). In female non-obese EFAD mice, APOE4 was associated with higher levels of $A D$ pathology; however the E3FAD mice were more affected by HFD, suggesting that the female E4FAD mice reached deficits that could not be further exacerbated by diet (Christensen and Pike, 2019). Therefore, while our study does not highlight diet specific behavioral deficits, data 
greatly support the connection between diet and CNS dysfunction.

Chronic systemic inflammation associated with VAT and the alterations in glucose and insulin may be connected to cognitive disturbances (Jones and Rebeck, 2018). HFD increases systemic inflammation from the increase in VAT (Hotamisligil et al., 1995). This increase in inflammation can both induce metabolic disturbances (Xu et al., 2003) and increase CNS damage (Kempuraj et al., 2017; Varatharaj and Galea, 2017). There is also the possibility that the metabolic disturbances such as glucose intolerance and insulin resistance could more directly lead to CNS damage. Metabolic disturbances have been associated with increased CNS insulin resistance, glucose intolerance (Arnold et al., 2014; Kothari et al., 2017), which can affect brain glucose uptake and neuronal functioning. However, we do not know whether it is the inflammation or metabolic disturbances leading the CNS deficits.

We found that HFD leads to metabolic disturbances particularly in male $A P O E 4$ mice, and in female mice of either APOE3 or APOE4 genotypes; however, the underlying mechanisms of this response remain to be defined. Overall, the study implicates APOE4 positive individuals as more affected by HFD. These connections could affect a large proportion of the population as the increasing rates of obesity increase the risk of metabolic syndrome.

\section{References}

Arbones-Mainar JM, Johnson LA, Altenburg MK, Maeda N (2008) Differential modulation of diet-induced obesity and adipocyte functionality by human apolipoprotein E3 and E4 in mice. Int $\mathrm{J}$ Obes (Lond) 32:1595-1605.

Arbones-Mainar JM, Johnson LA, Torres-Perez E, Garcia AE, PerezDiaz S, Raber J, Maeda N (2016) Metabolic shifts toward fatty-acid usage and increased thermogenesis are associated with impaired adipogenesis in mice expressing human APOE4. Int $\mathrm{J}$ Obes (Lond) 40:1574-1581.

Arnold SE, Lucki I, Brookshire BR, Carlson GC, Browne CA, Kazi H, Bang S, Choi BR, Chen Y, McMullen MF, Kim SF (2014) High fat diet produces brain insulin resistance, synaptodendritic abnormalities and altered behavior in mice. Neurobiol Dis 67:79-87.

Barron AM, Rosario ER, Elteriefi R, Pike CJ (2013) Sex-specific effects of high fat diet on indices of metabolic syndrome in 3xTg-AD mice: implications for Alzheimer's disease. PLoS One 8:e78554.

Besser LM, Gill DP, Monsell SE, Brenowitz W, Meranus DH, Kukull W, Gustafson DR (2014) Body mass index, weight change, and clinical progression in mild cognitive impairment and Alzheimer disease. Alzheimer Dis Assoc Disord 28:36-43.

Bloor ID, Symonds ME (2014) Sexual dimorphism in white and brown adipose tissue with obesity and inflammation. Horm Behav 66:95103.

Bour A, Grootendorst J, Vogel E, Kelche C, Dodart JC, Bales K, Moreau PH, Sullivan PM, Mathis C (2008) Middle-aged human apoE4 targeted-replacement mice show retention deficits on a wide range of spatial memory tasks. Behav Brain Res 193:174182.

Christensen A, Pike CJ (2019) APOE genotype affects metabolic and Alzheimer-related outcomes induced by Western diet in female EFAD mice. FASEB J 33:4054-4066.

Elias MF, Elias PK, Sullivan LM, Wolf PA, D'Agostino RB (2005) Obesity, diabetes and cognitive deficit: The Framingham Heart Study. Neurobiol Aging 26 Suppl 1:11-16.

Elosua R, Demissie S, Cupples LA, Meigs JB, Wilson PW, Schaefer EJ, Corella D, Ordovas JM (2003) Obesity modulates the associ- ation among APOE genotype, insulin, and glucose in men. Obes Res 11:1502-1508.

Enzinger C, Fazekas F, Matthews PM, Ropele S, Schmidt H, Smith S, Schmidt R (2005) Risk factors for progression of brain atrophy in aging: six-year follow-up of normal subjects. Neurology 64:17041711.

Ghebranious N, Mukesh B, Giampietro PF, Glurich I, Mickel SF, Waring SC, McCarty CA (2011) A pilot study of gene/gene and gene/environment interactions in Alzheimer disease. Clin Med Res 9:17-25.

Gunstad J, Paul RH, Cohen RA, Tate DF, Spitznagel MB, Gordon E (2007) Elevated body mass index is associated with executive dysfunction in otherwise healthy adults. Compr Psychiatry 48:5761.

Gustafson DR, Karlsson C, Skoog I, Rosengren L, Lissner L, Blennow K (2007) Mid-life adiposity factors relate to blood-brain barrier integrity in late life. J Intern Med 262:643-650.

Gustafson DR, Backman K, Waern M, Ostling S, Guo X, Zandi P, Mielke MM, Bengtsson C, Skoog I (2009) Adiposity indicators and dementia over 32 years in Sweden. Neurology 73:1559-1566.

Hadaegh F, Hasheminia M, Lotfaliany M, Mohebi R, Azizi F, Tohidi M (2013) Incidence of metabolic syndrome over 9 years follow-up; the importance of sex differences in the role of insulin resistance and other risk factors. PLoS One 8:e76304.

Hales CM, Carroll MD, Fryar CD, Ogden CL (2017) Prevalence of obesity among adults and youth: United States, 2015-2016. NCHS Data Brief 288:1-8.

Hotamisligil GS, Arner P, Caro JF, Atkinson RL, Spiegelman BM (1995) Increased adipose tissue expression of tumor necrosis factor-alpha in human obesity and insulin resistance. J Clin Invest 95:2409-2415

Huang Y, Mahley RW (2014) Apolipoprotein E: structure and function in lipid metabolism, neurobiology, and Alzheimer's diseases. Neurobiol Dis 72:3-12.

Huang Y, Weisgraber KH, Mucke L, Mahley RW (2004) Apolipoprotein $\mathrm{E}$ : diversity of cellular origins, structural and biophysical properties, and effects in Alzheimer's disease. J Mol Neurosci 23:189-204.

Huebbe P, Dose J, Schloesser A, Campbell G, Glüer CC, Gupta Y, Ibrahim S, Minihane AM, Baines JF, Nebel A, Rimbach G (2015) Apolipoprotein $\mathrm{E}$ (APOE) genotype regulates body weight and fatty acid utilization-Studies in gene-targeted replacement mice. Mol Nutr Food Res 59:334-343.

Hwang LL, Wang CH, Li TL, Chang SD, Lin LC, Chen CP, Chen CT, Liang KC, Ho IK, Yang WS, Chiou LC (2010) Sex differences in high-fat diet-induced obesity, metabolic alterations and learning, and synaptic plasticity deficits in mice. Obesity (Silver Spring) 18:463-469.

Janssen Cl, Jansen D, Mutsaers MP, Dederen PJ, Geenen B, Mulder MT, Kiliaan AJ (2016) The effect of a high-fat diet on brain plasticity, inflammation and cognition in female ApoE4-knockin and ApoE-knockout mice. PLoS One 11:e0155307.

Johnson LA, Torres ER, Impey S, Stevens JF, Raber J (2017) Apolipoprotein $\mathrm{E} 4$ and insulin resistance interact to impair cognition and alter the epigenome and metabolome. Sci Rep 7:43701.

Johnson LA, Torres ER, Weber Boutros S, Patel E, Akinyeke T, Alkayed NJ, Raber J (2019) Apolipoprotein E4 mediates insulin resistance-associated cerebrovascular dysfunction and the postprandial response. J Cereb Blood Flow Metab 39:770-781.

Jones NS, Rebeck GW (2018) The synergistic effects of APOE genotype and obesity on Alzheimer's disease risk. Int J Mol Sci 20:E63.

Kempuraj D, Thangavel R, Selvakumar GP, Zaheer S, Ahmed ME, Raikwar SP, Zahoor H, Saeed D, Natteru PA, lyer S, Zaheer A (2017) Brain and peripheral atypical inflammatory mediators potentiate neuroinflammation and neurodegeneration. Front Cell Neurosci 11:216.

Kesby JP, Kim JJ, Scadeng M, Woods G, Kado DM, Olefsky JM, Jeste DV, Achim CL, Semenova S (2015) Spatial cognition in adult and aged mice exposed to high-fat diet. PLoS One 10:e0140034. 
Knight EM, Martins IV, Gümüsgöz S, Allan SM, Lawrence CB (2014) High-fat diet-induced memory impairment in triple-transgenic Alzheimer's disease (3xTgAD) mice is independent of changes in amyloid and tau pathology. Neurobiol Aging 35:1821-1832.

Kothari V, Luo Y, Tornabene T, O'Neill AM, Greene MW, Geetha T, Babu JR (2017) High fat diet induces brain insulin resistance and cognitive impairment in mice. Biochim Biophys Acta Mol Basis Dis 1863:499-508.

Lin B, Hasegawa Y, Takane K, Koibuchi N, Cao C, Kim-Mitsuyama S (2016) High-fat-diet intake enhances cerebral amyloid angiopathy and cognitive impairment in a mouse model of Alzheimer's disease, independently of metabolic disorders. J Am Heart Assoc 5:e003154.

Liu CC, Liu CC, Kanekiyo T, Xu H, Bu G (2013) Apolipoprotein E and Alzheimer disease: risk, mechanisms and therapy. Nat Rev Neurol 9:106-118.

Macotela Y, Boucher J, Tran TT, Kahn CR (2009) Sex and depot differences in adipocyte insulin sensitivity and glucose metabolism. Diabetes 58:803-812.

Mathieu P, Poirier P, Pibarot P, Lemieux I, Després JP (2009) Visceral obesity: the link among inflammation, hypertension, and cardiovascular disease. Hypertension 53:577-584.

Medrikova D, Jilkova ZM, Bardova K, Janovska P, RossmeisI M, Kopecky J (2012) Sex differences during the course of dietinduced obesity in mice: adipose tissue expandability and glycemic control. Int J Obes (Lond) 36:262-272.

Moser VA, Pike CJ (2016) Obesity and sex interact in the regulation of Alzheimer's disease. Neurosci Biobehav Rev 67:102-118.

Moser VA, Pike CJ (2017) Obesity accelerates Alzheimer-related pathology in APOE4 but not APOE3 mice. eNeuro 4: ENEURO. 0077-17.2017.

Neth BJ, Craft S (2017) Insulin resistance and Alzheimer's disease: bioenergetic linkages. Front Aging Neurosci 9:345.

Neu SC, Pa J, Kukull W, Beekly D, Kuzma A, Gangadharan P, Wang LS, Romero K, Arneric SP, Redolfi A, Orlandi D, Frisoni GB, Au R, Devine S, Auerbach S, Espinosa A, Boada M, Ruiz A, Johnson SC, Koscik R, Wang JJ, at al. (2017) Apolipoprotein E genotype and sex risk factors for Alzheimer disease: a meta-analysis. JAMA Neurol 74:1178-1189.

Pradhan AD (2014) Sex differences in the metabolic syndrome: implications for cardiovascular health in women. Clin Chem 60: 44-52.

Profenno LA, Porsteinsson AP, Faraone SV (2010) Meta-analysis of Alzheimer's disease risk with obesity, diabetes, and related disorders. Biol Psychiatry 67:505-512.

Raber J, Huang Y, Ashford JW (2004) ApoE genotype accounts for the vast majority of $A D$ risk and $A D$ pathology. Neurobiol Aging 25:641-650.
Rodriguez GA, Burns MP, Weeber EJ, Rebeck GW (2013) Young APOE4 targeted replacement mice exhibit poor spatial learning and memory, with reduced dendritic spine density in the medial entorhinal cortex. Learn Mem 20:256-266.

Schulz TJ, Tseng YH (2013) Brown adipose tissue: development, metabolism and beyond. Biochem J 453:167-178.

Seibenhener ML, Wooten MC (2015) Use of the open field maze to measure locomotor and anxiety-like behavior in mice. J Vis Exp. Advance online publication. Retrieved February 6, 2015. doi: 10.3791/52434.

Speidell AP, Demby T, Lee Y, Rodriguez O, Albanese C, Mandelblatt J, Rebeck GW (2019) Development of a human APOE knock-in mouse model for study of cognitive function after cancer chemotherapy. Neurotox Res 35:291-303.

Tejedor MT, Garcia-Sobreviela MP, Ledesma M, Arbones-Mainar JM (2014) The apolipoprotein E polymorphism rs7412 associates with body fatness independently of plasma lipids in middle aged men. PLoS One 9:e108605.

To AW, Ribe EM, Chuang TT, Schroeder JE, Lovestone S (2011) The $\varepsilon 3$ and $\varepsilon 4$ alleles of human APOE differentially affect tau phosphorylation in hyperinsulinemic and pioglitazone treated mice. PLoS One 6:e16991.

Tolppanen AM, Ngandu T, Kåreholt I, Laatikainen T, Rusanen M, Soininen H, Kivipelto M (2014) Midlife and late-life body mass index and late-life dementia: results from a prospective population-based cohort. J Alzheimers Dis 38:201-209.

Torres-Perez E, Ledesma M, Garcia-Sobreviela MP, Leon-Latre M, Arbones-Mainar JM (2016) Apolipoprotein E4 association with metabolic syndrome depends on body fatness. Atherosclerosis 245:35-42.

Varatharaj A, Galea I (2017) The blood-brain barrier in systemic inflammation. Brain Behav Immun 60:1-12.

Whitmer RA, Gunderson EP, Barrett-Connor E, Quesenberry CP Jr, Yaffe K (2005) Obesity in middle age and future risk of dementia: a 27 year longitudinal population based study. BMJ 330:1360.

Whitmer RA, Gustafson DR, Barrett-Connor E, Haan MN, Gunderson EP, Yaffe K (2008) Central obesity and increased risk of dementia more than three decades later. Neurology 71:1057-1064.

Xu H, Barnes GT, Yang Q, Tan G, Yang D, Chou CJ, Sole J, Nichols A, Ross JS, Tartaglia LA, Chen H (2003) Chronic inflammation in fat plays a crucial role in the development of obesity-related insulin resistance. J Clin Invest 112:1821-1830.

Zade D, Beiser A, McGlinchey R, Au R, Seshadri S, Palumbo C, Wolf PA, DeCarli C, Milberg W (2013) Apolipoprotein epsilon 4 allele modifies waist-to-hip ratio effects on cognition and brain structure. J Stroke Cerebrovasc Dis 22:119-125. 